\title{
Preprocessing method of night vision image application in apple harvesting robot
}

\author{
Weikuan Jia ${ }^{1,3}$, Yuanjie Zheng ${ }^{1,4 *}$, De'an Zhao ${ }^{2}$, Xiang Yin ${ }^{3}$, Xiaoyang Liư ${ }^{2}$, Ruicheng Du $^{3}$ \\ (1. School of Information Science and Engineering, Shandong Normal University, Jinan 250358, China; \\ 2. Key Laboratory of Facility Agriculture Measurement and Control Technology and Equipment of Machinery Industry, \\ Jiangsu University, Zhenjiang 212013, China; \\ 3. School of Agricultural Engineering and Food Science, Shandong University of Technology, Zibo 255000, China; \\ 4. Institute of Life Sciences and Key Lab of Intelligent Information Processing, Shandong Normal University, Jinan 250014, China)
}

\begin{abstract}
Due to the low working efficiency of apple harvesting robots, there is still a long way to go for commercialization. The machine performance and extended operating time are the two research aspects for improving efficiencies of harvesting robots, this study focused on the extended operating time and proposed a round-the-clock operation mode. Due to the influences of light, temperature, humidity, etc., the working environment at night is relatively complex, and thus restricts the operating efficiency of the apple harvesting robot. Three different artificial light sources (incandescent lamp, fluorescent lamp, and LED lights) were selected for auxiliary light according to certain rules so that the apple night vision images could be captured. In addition, by color analysis, night and natural light images were compared to find out the color characteristics of the night vision images, and intuitive visual and difference image methods were used to analyze the noise characteristics. The results showed that the incandescent lamp is the best artificial auxiliary light for apple harvesting robots working at night, and the type of noise contained in apple night vision images is Gaussian noise mixed with some salt and pepper noise. The preprocessing method can provide a theoretical and technical reference for subsequent image processing.
\end{abstract}

Keywords: apple harvesting robot, night vision image, preprocessing method, color analysis, noise analysis DOI: $10.25165 / \mathrm{j}$.jjabe.20181102.2822

Citation: Jia W K, Zheng Y J, Zhao D A, Yin X, Liu X Y, Du R C. Preprocessing method of night vision image application in apple harvesting robot. Int J Agric \& Biol Eng, 2018; 11(2): 158-163.

\section{Introduction}

According to the statistics of the Food and Agriculture Organization (FAO) of the United Nations, apples are ranked second in global fruit production. These statistics also show that China is the largest apple producer with an apple planting area and production exceeding $50 \%$ of the world and keeping an increasing tend during recent years. In the whole process of apple production, harvesting work is time-consuming and laborious and has strong seasonality. At present, apple harvesting is usually completed by manual operation, which is a highly labor-intensive task. In addition, the urbanization and gradual population aging also cause an acute shortage of agricultural labor in recent years. All the above factors restrict apple production and contribute to losses in agricultural production. To alleviate these contradictions,

Received date: 2017-06-06 Accepted date: 2018-02-08

Biographies: Weikuan Jia, PhD, Lecturer, research interests: agricultural information, artificial intelligence, Email: jwk_1982@163.com; De'an Zhao, $\mathrm{PhD}$, Professor, research interests: agricultural robots, intelligent control, Email: dazhao@ujs.edu.cn; Xiang Yin, PhD, Lecturer, research interests: agricultural information, navigation control, Email: yinxiang2013@yahoo.co.jp; Xiaoyang Liu, PhD candidate, research interests: agricultural robots, agricultural information, Email: 774294228@qq.com; Ruicheng Du, Professor, research interests: agricultural machinery, Email: drc@sdut.edu.cn.

*Corresponding author: Yuanjie Zheng, PhD, Professor, research interests: intelligent information processing, artificial intelligence. School of Information Science and Engineering, Engineering, Shandong Normal University, No.1, Daxue Road, Changqing District, Jinan 250358, China. Email: yjzheng@ sdnu.edu.cn. fortunately, fruit and vegetable harvesting robot technology is improving.

Nowadays, agricultural robots ${ }^{[1,2]}$ or harvesting robots ${ }^{[3-7]}$ have reached a new level. Moreover, the related studies of apple harvesting robot have also been carried out gradually and achieved good results ${ }^{[8-10]}$. For example, AFPM, one type of apple harvesting robot that was developed by a Belgian scholar for apple sizes of 6-11 cm in diameter, has a harvesting rate of approximately $80 \%$ and a harvest cycle of approximately $9 \mathrm{~s}$, which is the general development level in the current agricultural world ${ }^{[8]}$. In China, the new generation prototype was co-developed by the Chinese Academy of Agricultural Machinery Sciences and Jiangsu University; under laboratory conditions, the harvesting rate also reaches $80 \%$ and the harvesting speed is $15 \mathrm{~s}$ per apple, which represents the highest level achieved ${ }^{[9]}$. Currently, due to the lower harvesting efficiency, the apple harvesting robot is still in the laboratory research stage. In general, there are two factors for improving harvesting efficiency under the unstructured environment of an orchard. One is the self-performance improvement of apple harvesting robots, such as the monocular vision $^{[11]}$ and binocular vision ${ }^{[12]}$, recognition and tracking of dynamic fruit ${ }^{[13]}$, manipulator obstacle avoidance ${ }^{[14]}$, path planning $^{[15]}$, green apple recognintion ${ }^{[16]}$, and so on. Another one is longer working time to implement the round-the-clock operation.

The key problem of night working is how to capture and deal with the apple night vision image. Related studies of this subject are few; however, with the help of many scholars, some progress has been attained in recent years. Payne captures night vision images of mangos which are nearly mature enough, and the number 
of mangos are recognized by color and texture features of $\mathrm{YCbCr}$ color space and then used to estimate the mango production ${ }^{[17]}$. Font acquires the night vision images of ripe grapes under GRB color space to determine the number of grapes by calculating the spherical reflection peak on the grape surface ${ }^{[18]}$. Guo et al. ${ }^{[19]}$ and Hayashi et al. ${ }^{[20]}$ applied artificial light to the vision system of strawberry harvesting robots to reduce the influence of ambient light and to improve the quality of the captured images. Zhang et al. ${ }^{[21]}$ use night vision images to design a kind of hybrid classifier which is composed of SVM and super green operator, enabling green apples to be effectively recognized against a similar color background. Liu et al. ${ }^{[22]}$ used fragmentation theory to recognize night vision images. Jia et al. ${ }^{[23]}$ studied the denoising and enhancing of apple night vision images. All of these provide a good theoretical basis for enabling night working of apple harvesting robots and provide promise for night working studies. Compared with a day working, the environment of night working is relatively complex to test the harvesting efficiency of apple harvesting robots. At night, many factors, such as light, temperature, humidity, etc., will restrict the quality of apple night images and influence the recognition precision of target fruits.

To better adapting to the night working, this paper focused on the research of apple night vision images for improving night working efficiency. First, in the apple orchard, different artificial lights were selected as auxiliary light, certain sampling rules were adopted, and a series of apple night vision images were captured. Second, comparing different images by color analysis, the appropriate artificial auxiliary light was selected by color characteristics of night vision images. Finally, the noise of each night vision image was analyzed, and the noise type was determined by visual observation and the difference images method, which can provide better theoretical support for night vision image de-noising.

\section{Materials and methods}

\subsection{Materials}

To achieve the round-the-clock working of apple harvesting robots, the continuous working under the environment of the night or lacking natural light should be realized. Night working needs to be done under the artificial auxiliary light when capturing the experimental apple images, and natural light and night vision imaging should both be considered. In this study, under different artificial lights, apple night vision images are captured.

\subsubsection{Artificial auxiliary light}

To make sure that the apple can be harvested at night, auxiliary lighting is the first consideration. An artificial auxiliary light source is easy to be controlled and has been widely used in industrial and agricultural production, as well as in our daily lives. Nevertheless, compared with natural light, artificial light has many deficiencies, such as low light intensity, uneven light, small illumination range and poor stability. Low-illumination environment will influence the acquisition quality of night image. Compared with natural light images, the captured night vision images have some differences and affect the efficiency of image processing.

Artificial light is a light-emitting device which can simulate the solar spectrum, and has the biggest advantage of controllability. In this study, three different artificial lights were selected, including incandescent lamps, fluorescent lamps and LED lights. Their color temperature and rendering have a great influence on night vision images. The color temperature of incandescent lamps is approximately $2700 \mathrm{~K}$, belonging to the warm-color light, and the color rendering is approximately 100. The fluorescent lamps and the LED's color temperatures are larger than $5000 \mathrm{~K}$, belonging to cool-color light, and the color rendering is between 70-80. The advantages and disadvantages of the three kinds of artificial lights are listed in Table 1.

Table 1 Comparison of the advantages and disadvantages of three types of artificial light

\begin{tabular}{cl}
\hline Types of artificial light & \multicolumn{1}{c}{ Working principle } \\
\hline \multirow{2}{*}{ Incandescent lamp } & $\begin{array}{l}\text { Through the current reaches above } 2000{ }^{\circ} \mathrm{C} \text { high } \\
\text { temperature to make the tungsten light in a vacuum } \\
\text { bulb or tube. }\end{array}$ \\
& $\begin{array}{l}\text { Through low-pressure gas to discharge lamp, bulb or } \\
\text { glass tube inner surface coated with phosphor, which } \\
\text { filled with mercury vapour and inert gas. }\end{array}$ \\
Fluorescent lamp & $\begin{array}{l}\text { A light emitting diode semiconductor devices, which } \\
\text { can convert electric power into visible light's solid } \\
\text { state. }\end{array}$ \\
\hline
\end{tabular}

In the apple orchard, the working environment of apple harvesting robots is outside, so when images are captured, the power source of artificial light is provided by the accumulator. In this study, the type of power source and artificial lights are as follows: lead-acid battery, $12 \mathrm{~V}$ DC output voltage, $36 \mathrm{~A} \cdot \mathrm{h}$ capacity; the rated voltage of Incandescent lamps is $12 \mathrm{~V} \mathrm{DC}$, power is $35 \mathrm{~W}$; fluorescent lamp, $12 \mathrm{~V} \mathrm{DC}, 35 \mathrm{~W}$; The LED light is made up of 9 groups of $1 \mathrm{~W}$ lights, which have aluminum plates.

Following the principle of shadowless lamps, auxiliary lighting equipment is designed to eliminate the shadow influence of captured images as much as possible. It is composed of 8 group lamps in a circle, with the camera located in the centre. Considering that the auxiliary light needs to be assembled on the apple harvesting robot, the circular radius should not be too large, with a stent length radius of no more than $0.5 \mathrm{~m}$, as shown in Figure 1.

Disadvantages
Using life is short, low
photosynthetic efficiency, send
out a lot of heat.
Poor stability and low power
factor.
low surface temperature.

Small size, without reducing illuminance, good Using life is short, low color rendering, low price, the instantaneous start, good stability.
Luminous high purity, high photoelectric conversion rate, good color rendering, does not need high pressure, no radiation, no pollution.
Low brightness values, Divergent light, high price.

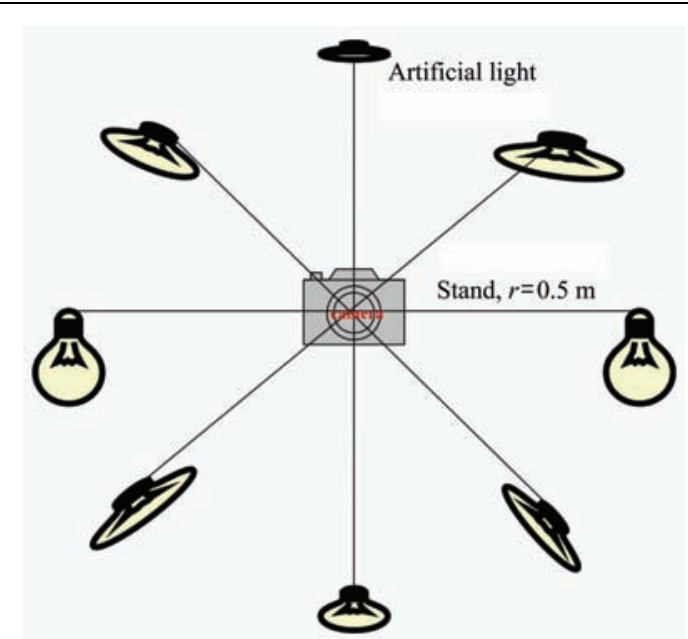

Figure 1 Design of service lighting 


\subsubsection{Captured environment}

Captured location: Dashahe apple production park, Fengxian, Jiangsu Province, China (experimental base of apple harvesting robot, Jiangsu University), apple plantations in Anxiangyujia village, Penglai, Shandong province, China.

Captured environment: natural light luminance is tested by a luminance tester (type: TASI-8720, the maximum range of $200000 \mathrm{~lx}$, the resolution is $1 \mathrm{~lx}$, Suzhou TASI Electronic Co., Ltd), the natural light luminance is below 1, artificial light is used for auxiliary lighting, and night vision images are captured.

Camera: AFT-0814MP, focal length $8 \mathrm{~mm}$, viewing Angle 54 degrees, relative optical path F1.4-C, image surface sizes 1/2", minimum object distance $0.15 \mathrm{~m}$.

Captured image method: select fruit sample points and then mark the location and direction of the fruits by benchmarking, keeping the same position and angle when the image is captured to make sure the apple image is consistent under the natural light and

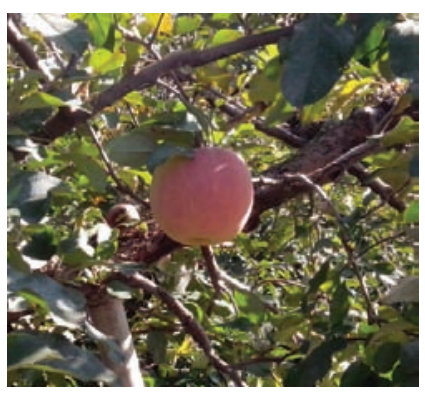

a1. Natural light

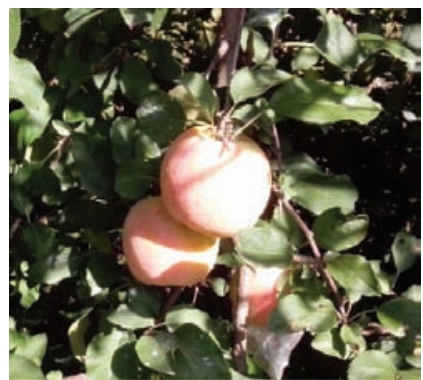

b1. Natural light

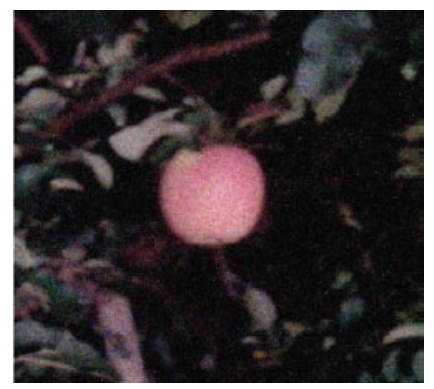

a2. Incandescent lamp

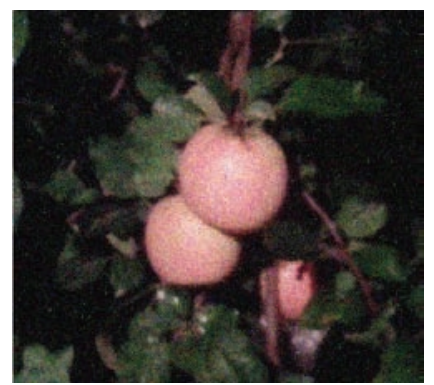

b2. Incandescent lamp

auxiliary lights.

Sample points of the image captured: the selection of fruit sample points should be representative. In practice, the eternally changing natural apple growth posture and the varied capture angles caused by different camera positions often make the captured fruit images showing a diversity of postures. This phenomenon is more evident in single fruits without sunscreen, overlapping fruit, fruits that are covered by leaves. Therefore, the sampling should be targeted and representative for achieving reasonable results.

\subsubsection{Night vision image}

To sum up, the apple images are captured from selected sample points. In this paper, an example of two groups of apple images from two sample points is shown in Figure 2. Under the same capture angle and position and different light environments, the groups of apple images are captured with natural light, incandescent lamp, fluorescent lamp and LED light.

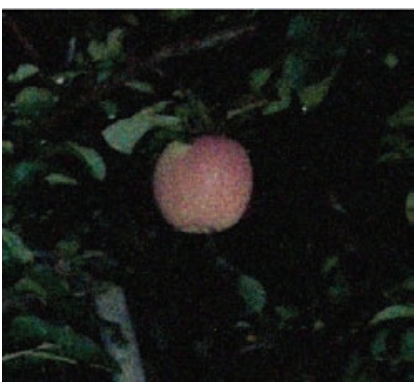

a3. Fluorescent lamp

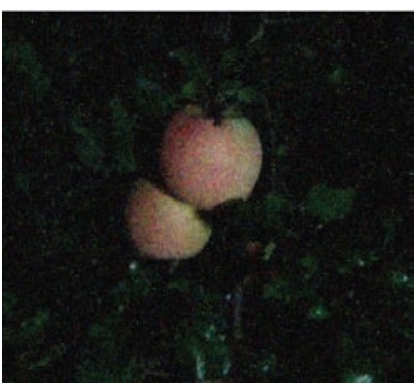

b3. Fluorescent lamp

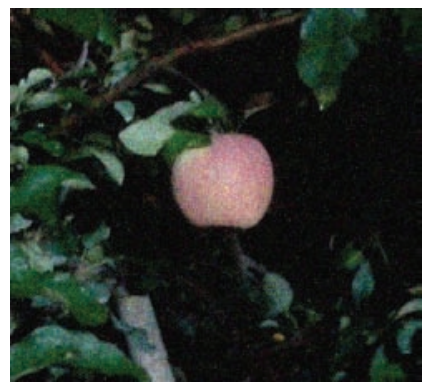

a4. LED

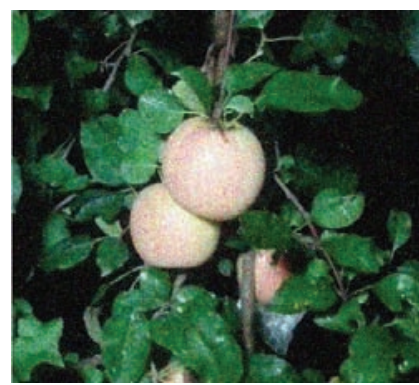

b4. LED

Figure 2 Apple original images under natural light and different artificial light sources

From Figure 2, we can clearly see that the night vision images are different from the natural light images, and the backgrounds of apple night vision images exhibit large dark space. On the whole, night vision images seem obscure, branches and leaves are not clear, and to a certain extent, the surfaces of target fruits highlight reflective area and shadow, and the edge of target fruits seems slightly obscured.

\subsection{Methods}

To a great extent, many factors will influence the quality of apple night vision images, including some characteristics of artificial light, such as illumination intensity, color temperature, color rendering, and so on, as well as some conditions of the night working, such as temperature, humidity, and so on. To further understand the apple night vision images, know their characteristics, and get ready for further processing, some analyses need to be done for night vision images, including color analysis, intuitive visual analysis, and difference image analysis. The main work of this study includes apple night vision image precise captured and preprocessing. The flowchart of night vision image preprocessing is shown in Figure 3.

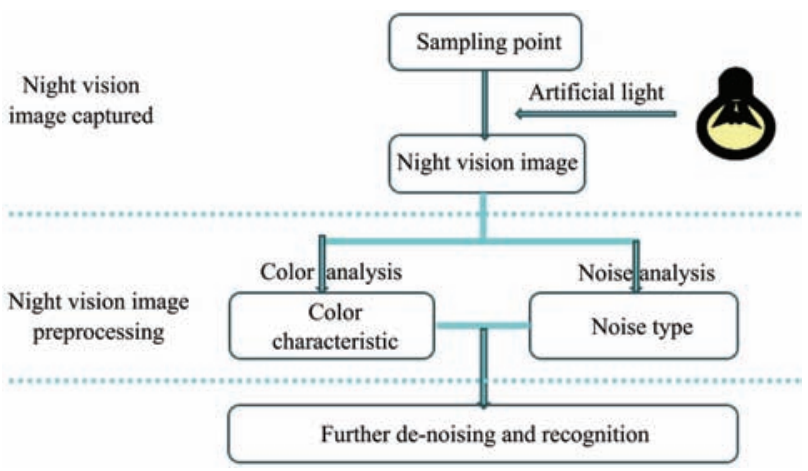

Figure 3 Flow chart of night vision image preprocessing

\subsubsection{Color analysis}

To analyze the color features corresponding to the different scenery in the captured apple images, four images of sample point, for example, were taken under the RGB color space, with color information decomposition of apple images.

\subsubsection{Intuitive visual analysis}

From Figure 2, compared with the apple images of different light environments, it seems obvious that night vision images under 
different auxiliary lights are obscure due to the existence of noise. Since artificial light having more deficiencies than natural light, and coupled with the relatively low temperature and high humidity in the night environment (factors that will influence the normal work of the image sensor), it is easy for the camera's exposure to be insufficient and lead to noise in apple night vision images.

To contrast the differences between captured apple images under different light environments more clearly, eight apple images were processed. They were transformed into grayscale mode, so the noisy points in images can be directly observed.

\subsubsection{Difference image analysis}

In the process of capturing apple night vision images, the camera's precision, the lighting environment provided by artificial auxiliary light, temperature and humidity of the surrounding environment, and even the change of ambient air may be leading to the noise in the night vision images. This noise will affect further processing of night vision images. First, the type of the noise needs to be determined, and then an appropriate de-noising
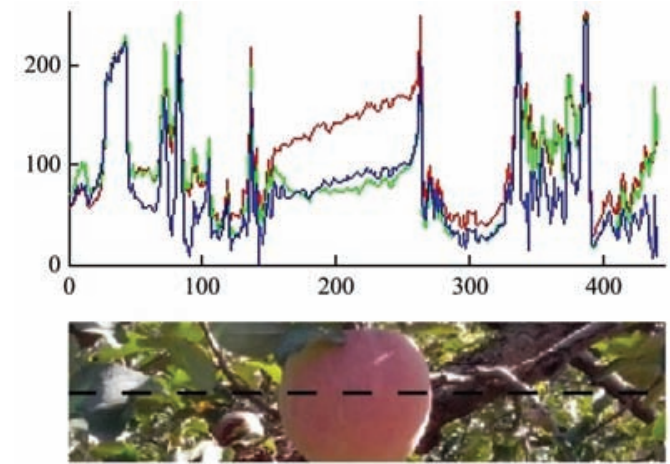

a. Color distribution of natural light image

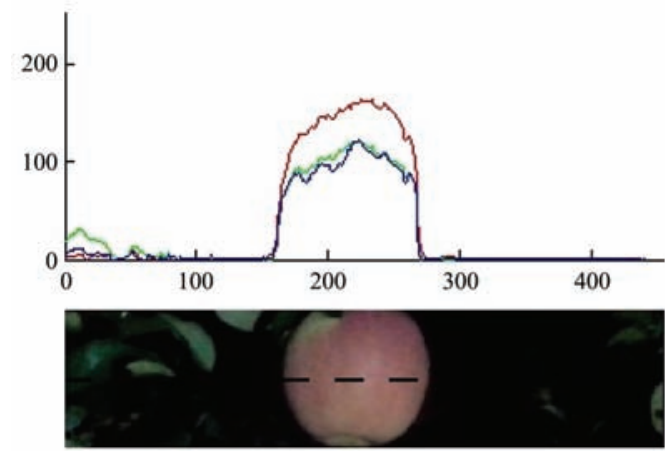

c. Color distribution of fluorescent lamp image algorithm is designed, to better eliminate noise.

In many existing studies related to night vision image research $^{[24,25]}$, Gaussian noise is the main noise of night vision images. Moreover, Gaussian noise commonly exists in signals of image, sound, and electromagnetism. To further determine the noise type of apple night vision images, this study attempts to introduce a different method for image analysis. The basic principle of this method is that for the same image capture environment and one sample point, the noise is generated randomly; if two same-size and static images are captured and if no noise exists, the gray value corresponding to the two static images subtraction is 0 ; if noise exists, it is impossible for the gray value subtraction to be 0 , and the subtraction image is the noise data.

\section{Results and discussion}

\subsection{Results of color analysis}

The color information decomposition of apple images figure $2 \mathrm{a}$ as shown in Figure 4.
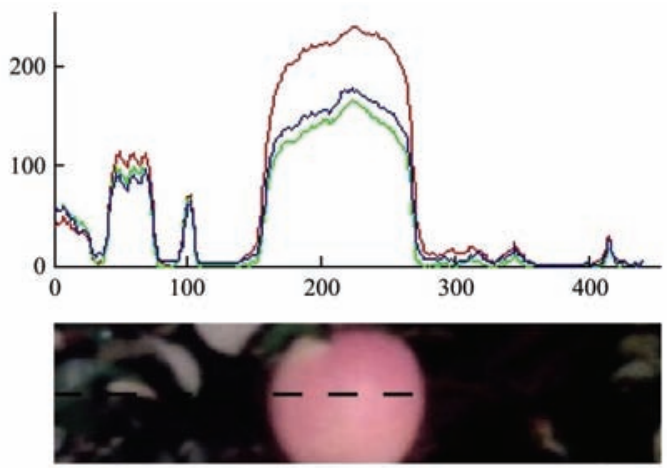

b. Color distribution of incandescent lamp image

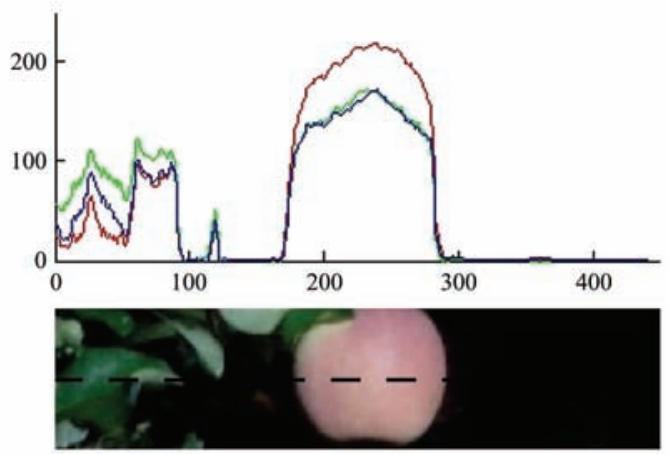

d. Color distribution of the LED image

Note: The red line represents the R component, the green line represents the G component, and the blue line represents the B component; these three lines show the black horizontal dotted line corresponding to RGB components of the image pixel.

Figure 4 Corresponding RGB components of images under different lights

In Figure 4, RGB components correspond to the different scenery in the apple natural light images and three different night vision images, and there are obvious differences of color features between natural-light images and night vision images. Comparing these night vision images in terms of color performance, it is discovered that the captured image has the highest color saturation under the incandescent lamps, the fluorescent lamp image is intermediate, and the color of the LED image is the coolest with the worst color saturation. Further comparing the RGB components of different landscapes, the advantage of the $R$ component of the image of the incandescent lamp is more obvious, and the $R$ component superiority of the other two kinds of night vision images is less obvious.

Comparing natural-light images and night vision images, the $R$ component of target fruits is obviously bigger than $G$ and $B$ components. For natural light images, the RGB component difference of leaves and benches is bigger, and this is mainly due to the attenuation characteristics of the light source. Otherwise, for night vision images, this difference is relatively smaller.

Overall, the color performance of apple night vision images under the incandescent lamp is closer to natural light images. From the perspective of the color feature, based on the advantage of night vision images under incandescent lamps, with incandescent lamps as the selected auxiliary light, it is more appropriate for apple harvesting robots to work at night.

\subsection{Intuitive visual analysis}

The transformed grayscale images are shown in Figure 5. 


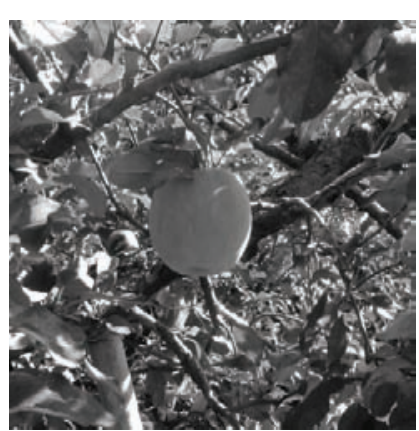

a1. Natural light

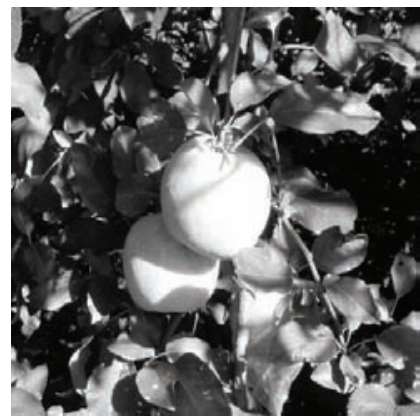

b1. Natural light

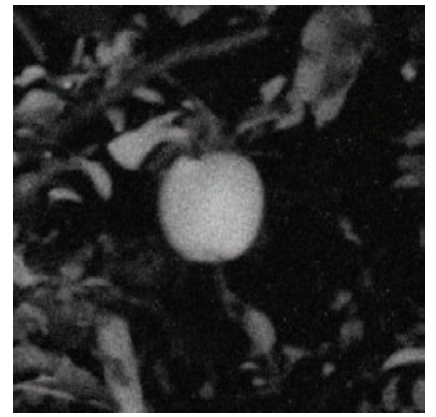

a2. Incandescent lamp

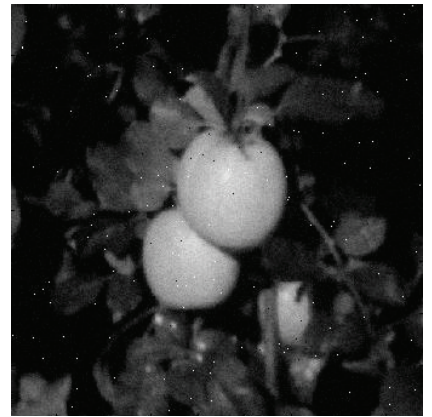

b2. Incandescent lamp

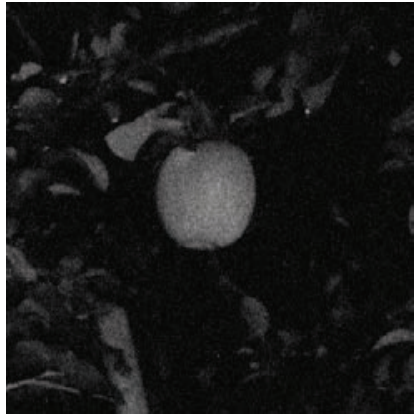

a3. Fluorescent lamp

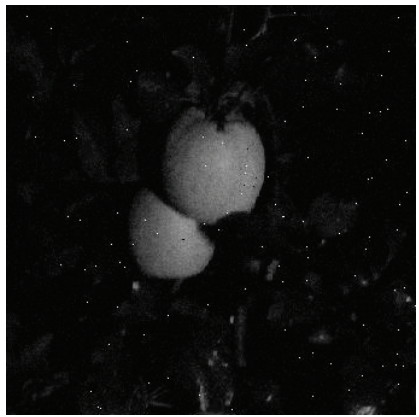

b3. Fluorescent lamp

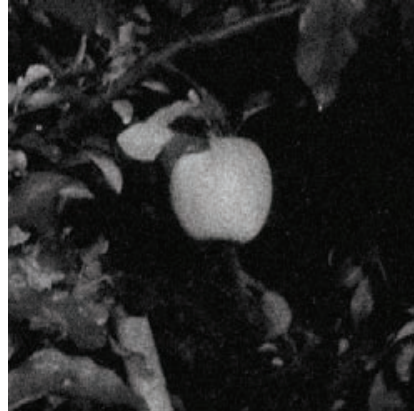

a4. LED

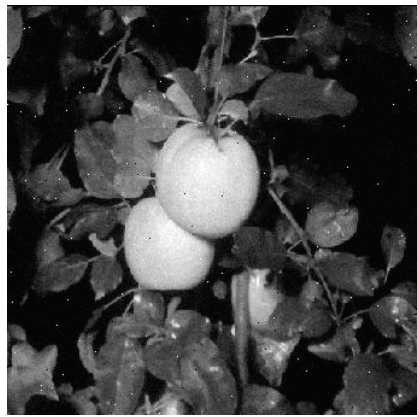

b4. LED

Figure 5 Grayscale of original apple images

From an intuitive visual point of view under grayscale mode, Figure 5 shows that compared with natural light images, three kinds of different night vision images contain relative amounts of noisy points. Preliminarily, these night vision images are suspected for mixed noise. Apple night vision images contain some salt and pepper noise; this noise type can be determined by visual observation directly, but other types can't be determined directly and need further analysis.

\subsection{Difference image analysis}

Here, the captured apple night vision image under an incandescent lamp is used as an example, in which the noise analysis is performed by the difference image algorithm. Under the same capture environment, two static apple night vision images are consecutively captured, and the image subtraction effect is shown in Figure 6.

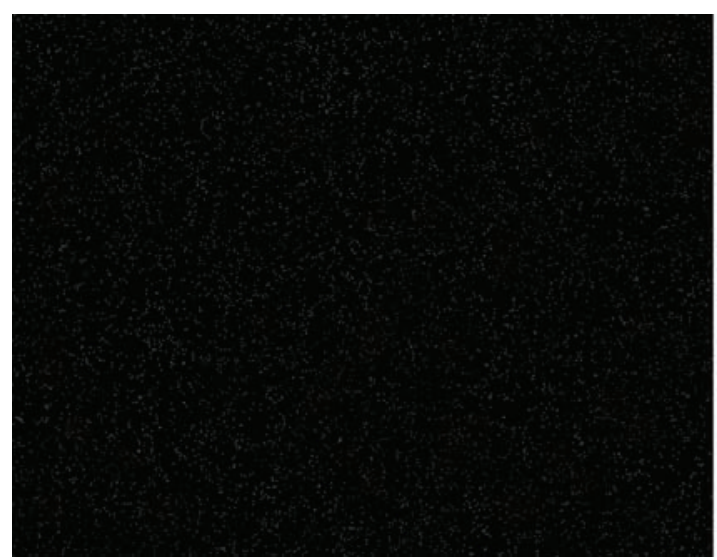

a. Noise image

Figure 6 Diagram of images subtraction
The subtraction of two night vision images by using the difference image method is shown in Figure 6a, which is computed by using the noise data of apple night vision images. The gray histogram of Figure $6 \mathrm{a}$ is shown in Figure $6 \mathrm{~b}$ to clarify that the gray histogram of noise data is close to the Gaussian distribution. Therefore, it was determined that this noise type is Gaussian noise.

Similarly, for the night vision image under two other kinds of different artificial auxiliary light, difference image analysis was completed and produced the same conclusion that the noise type is Gaussian noise. In addition, for multiple sets of sample points, the results of repeated testing also led to the same conclusion.

Summing up the above, in this study, the noise type of several apple night vision images under different artificial auxiliary light is mixed noise, in which Gaussian noise is given priority, with some salt and pepper noise.

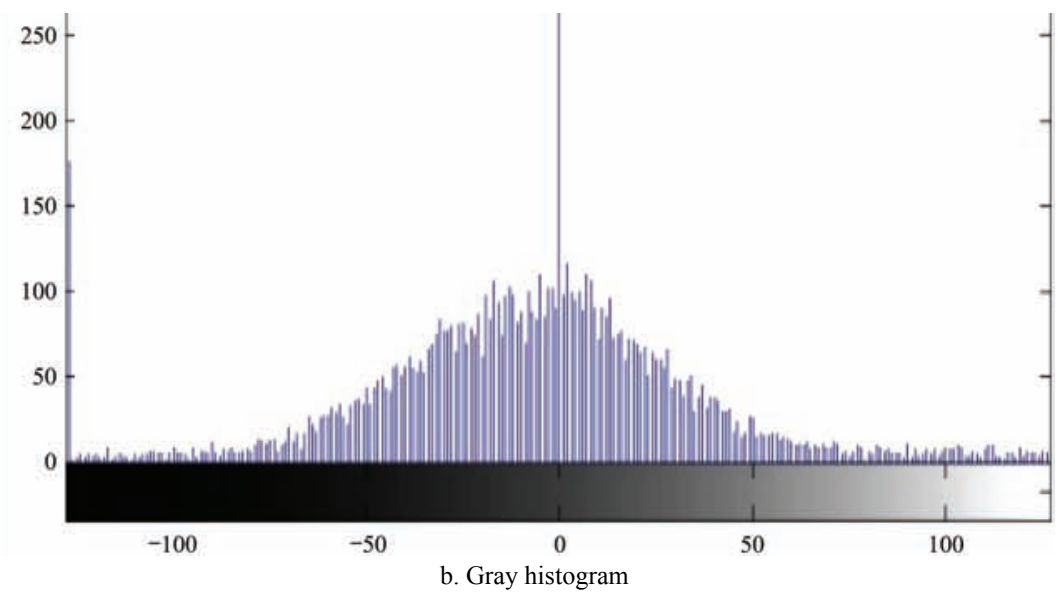

\subsection{Discussion}

This study focused on three aspects: the acquisition of night vision images, color analysis of natural and night vision images, and noise analysis for night vision images. Since the night working environment is very complex, and many factors can influence the quality of night vision images, such as the distance between target and light source, exposure time, the power of the artificial light source, and so on. To better process night vision images, further work should focus on getting more accurate image information, providing the precision signal for target recognition ${ }^{[26]}$, video or image processing ${ }^{[27,28]}$, and servo control ${ }^{[29]}$, etc. 


\section{Conclusions}

To achieve the round-the-clock working of apple harvesting robots, the night vision image is regarded as the research object of this study. Focusing on images captured at night, the performance, cost and practicability of artificial light, incandescent lamps, fluorescent lamps, and LED lamps were considered and used to fill light. According to the set conditions, the images were captured, and natural light and night vision images of the same sample points were obtained. Some related analyses of these images were required to better understand differences.

By color analysis, it has been found that in both natural light and night vision images, the $R$ component of target fruits is bigger than $G$ and $B$ components. For color component differences between the branches or leaves, natural light images reveal bigger differences than night vision images; the comparison of three kinds of night vision images, the color feature of incandescent lamp images was found to be closer to natural light images and had the highest color saturation. From the intuitive visual observations, compared with natural light images, apple night vision images are relatively obscure - the edge of the target fruit is not clear, and the night vision image contains much noise including salt and pepper noise. To further determine the noise type, this study uses night vision images under the incandescent lamp as an example, difference image analysis is introduced, and the results show that night vision images contain lots of Gaussian noises. Two other kinds of night vision images are tested by difference image analysis, and when the experiment was repeated, the results are consistent. Therefore, the noise type of the night vision image is determined as mixed noise, in which Gaussian noise is given priority with some salt and pepper noise.

\section{Acknowledgments}

This work was financially supported by the Natural Science Foundation of Shandong Province in China (ZR2017BC013, ZR2014FM001); National Nature Science Foundation of China (No. 31571571, 61572300); Taishan Scholar Program of Shandong Province of China (No.: TSHW201502038); and Priority Academic Program Development of Jiangsu Higher Education Institutions.

\section{[References]}

[1] Pedersen S M, Fountas S, Have H, Blackmore B S. Agricultural robots system analysis and economic feasibility. Precision agriculture, 2006; 7(4): 295-308.

[2] Zhang L B, Yang Q H, Bao G J, Wang Y, Qi L Y, Gao F, et al. Overview of research on agricultural robots in China. Int J Agric \& Biol Eng, 2008; 1(1): 12-21.

[3] Van Henten E J, Van't Slot D A, Hol C W J, van Willigenburg L G. Optimal manipulator design for a cucumber harvesting robot. Computers and Electronics in Agriculture, 2009; 65(2): 247-257.

[4] Bac C W, Van Henten E J, Hemming J, Edan Y. Harvesting robots for high-value crops: state-of-the-art review and challenges ahead. Journal of Field Robotics, 2014; 31(6): 888-911.

[5] Fernández R, Salinas C, Montes H, Sarria J. Multisensory system for fruit harvesting robots. Experimental testing in natural scenarios and with different kinds of crops. Sensors, 2014; 14(12): 23885-23904.

[6] Wang L L, Zhao B, Fan J W, Hu X A, Wei S, Li Y S, et al. Development of a tomato harvesting robot used in greenhouse. Int J Agric \& Biol Eng, 2017; 10(4): 140-149.

[7] Feng Q C, Zou W, Fan P F, Zhang C F, Wang X. Design and test of robotic harvesting system for cherry tomato. Int J Agric \& Biol Eng,
2018; 11(1): 96-100.

[8] Baeten J, Donné K, Boedrij S, Beckers W, Claesen E. Autonomous fruit picking machine: a robotic apple harvester. Springer Tracts in Advanced Robotics, 2008; 42: 531-539.

[9] Zhao D A, Lv J D, Ji W, Zhang Y, Chen Y. Design and control of an apple harvesting robot. Biosystems Engineering, 2011; 110(2): 112-122.

[10] Ji W, Zhao D A, Cheng F Y, Xu B, Zhang Y, Wang J J. Automatic recognition vision system guided for apple harvesting robot. Computers and Electrical Engineering, 2012; 38(5): 1186-1195.

[11] Xiao C Y, Zheng L H, Li M Z, Ma C Y. Apple detection from apple tree image based on BP neural network and Hough transform. Int J Agric \& Biol Eng, 2015; 8(6): 46-53.

[12] Cai J R, Sun H B, Li Y P, Sun L, Lu H. Fruit trees 3-D information perception and reconstruction based on binocular stereo vision. Transactions of the CSAM, 2012; 43(3): 152-156. (in Chinese)

[13] Zhao D A, Shen T, Chen Y, Jia W K. Fast tracking and recognition of overlapping fruit for apple harvesting robot. Transactions of the CSAE, 2015; 31(2): 22-28. (in Chinese)

[14] Ji W, Cheng F Y, Zhao D A, Lü J. Obstacle avoidance method of apple harvesting robot manipulator. Transactions of the CSAM, 2013; 44(11): 253-259. (in Chinese)

[15] Yuan Y W, Zhang X C, Hu X A. Algorithm for optimization of apple harvesting path and simulation. Transactions of the CSAE, 2009; 25(4): 141-144. (in Chinese)

[16] Li B R, Long Y, Song H B. Detection of green apples in natural scenes based on saliency theory and Gaussian curve fitting. Int J Agric \& Biol Eng, 2018; 11(1): 192-198.

[17] Payne A B, Walsh K B, Subedi P P, Jarvis D. Estimating mango crop yield using image analysis using fruit at 'stone hardening' stage and night time imaging. Computers and Electronics in Agriculture, 2014; 100: $160-167$.

[18] Font $\mathrm{D}$, Pallejà $\mathrm{T}$, Tresanchez $\mathrm{M}$, Teixidó $\mathrm{M}$, Martinez $\mathrm{D}$, Moreno $\mathrm{J}$, et al Counting red grapes in vineyards by detecting specular spherical reflection peaks in RGB images obtained at night with artificial illumination. Computers and Electronics in Agriculture, 2014; 108: 105-111.

[19] Guo F, Cao Q X, Cui Y J, Masateru N. Fruit location and stem detection method for strawberry harvesting robot. Transactions of the CSAE, 2008; 24(10): 89-94.

[20] Hayashi S, Shigematsu K, Yamamoto S, Kurita M. Evaluation of a strawberry-harvesting robot in a field test. Biosystems Engineering, 2010, 105: 160-171.

[21] Zhang C L, Zhang J, Zhang J X, Li W. Recognition of green apple in similar background. Transactions of the CSAM, 2014; 45(10): 277-281. (in Chinese).

[22] Liu X Y, Zhao D A, Jia W K, Ruan C Z, Tang S P, Shen T. A method of segmenting apples at night based on color and position information. Computers and Electronics in Agriculture, 2016; 122: 118-123.

[23] Jia W K, Zhao D A, Ruan C Z, Liu X Y, Chen Y Y, Ji W. Combination method of night vision image denoising based on wavelet transform and ICA. Transactions of the CSAM, 2015; 46(9): 9-17. (in Chinese)

[24] Han J, Yue J, Zhang Y, Bai L F. Salient contour extraction from complex natural scene in night vision image. Infrared Physics \& Technology, 2014; 63: $165-177$.

[25] Talebi H, Milanfar P. Global image denoising. IEEE Transactions on Image Processing, 2014; 23(2): 755-768.

[26] Zhao M, Zhang H, Sun J. A novel image retrieval method based on multi-trend structure descriptor. Journal of Visual Communication \& Image Representation, 2016; 38(C): 73-81.

[27] Hou S J, Chen L, Tao D C, Zheng Y J. Multi-layer multi-view topic model for classifying advertising video. Pattern Recognition, 2017; 68: 66-81.

[28] Meng L L, Liang J, Samarawickrama U, Zhao Y, Bai H H, Kaup A. Multiple description coding with randomly and uniformly offset quantizers. IEEE Transactions on Image Processing, 2014, 23(2): 582-595.

[29] Ding S H, Levant A, Li S H. Simple homogeneous sliding-mode controller. Automatica, 2016; 67: 22-32.

[30] Liu X M, Zhang K J, Li S T, Wei H K. Optimal control of switching times in switched stochastic systems. Asian Journal of Control, 2015; 17(5): 1580-1589. 